\title{
Uncoupled thermoelasticity solutions applied on beam dumps
}

\author{
A. Ouzia ${ }^{1}$ and T. Antonakakis ${ }^{2}$ \\ ${ }^{1}$ UPMC Univ Paris 06, Département de mathématiques, F-75005 Paris, France \\ ${ }^{2}$ Multiwave Technologies AG, 1228 Geneva, Switzerland \\ (Received 29 January 2015; published 13 June 2016)
}

\begin{abstract}
In particle accelerators the process of beam absorption is vital. At CERN particle beams are accelerated at energies of the order of $\mathrm{TeV}$. In the event of a system failure or following collisions, the beam needs to be safely absorbed by dedicated protecting blocks. The thermal shock caused by the rapid energy deposition within the absorbing block causes thermal stresses that may rise above critical levels. The present paper provides a convenient expression of such stresses under hypotheses described hereafter. The temperature field caused by the beam energy deposition is assumed to be Gaussian. Such a field models a non-diffusive heat deposition. These effects are described as thermoelastic as long as the stresses remain below the proportional limit and can be analytically modeled by the coupled equations of thermoelasticity. The analytical solution to the uncoupled thermoelastic problem in an infinite domain is presented herein and matched with a finite unit radius sphere. The assumption of zero diffusion as well as the validity of the match with a finite geometry is quantified such that the obtained solutions can be rigorously applied to real problems. Furthermore, truncated series solutions, which are not novel, are used for comparison purposes. All quantities are nondimensional and the problem reduces to a dependence of five dimensionless parameters. The equations of elasticity are presented in the potential formulation where the shear potential is assumed to be nil due to the source being a gradient and the absence of boundaries. Nevertheless equivalent three-dimensional stresses are computed using the compressive potential and optimized using standard analytical optimization methods. An alternative algorithm for finding the critical points of the three-dimensional stress function is presented. Finally, a case study concerning the proton synchrotron booster dump is presented where the aforementioned analytical solutions are used and the preceding assumptions verified.
\end{abstract}

DOI: 10.1103/PhysRevAccelBeams.19.063501

\section{INTRODUCTION}

Beam dumps and beam stoppers are blocks of material dedicated to absorbing charged particles and consequently removing them from circulating beams. Typical issues arising from with the design of a beam dump include: heat dissipation, radioactivation, radiation damages and thermal stresses on which we focus herein. For beam energies in $\mathrm{GeV}$ or $\mathrm{TeV}$ range as in the proton synchrotron booster (PSB) and LHC respectively, the physics involved in the energy deposited is governed by the electromagnetic shower development initiated by nuclear reactions of beam particles impacting the material lattice [1]. FLUKA [2,3], a Monte-Carlo based software, is currently the standard tool used at CERN for this important application.

In the context of the proton synchrotron booster used to inject the beam from the linear accelerators to the proton synchrotron (PS) [4,5], it is essential to ensure the interception of high energy proton beams. This is accomplished by using beam intercepting devices (BIDs) where the

Published by the American Physical Society under the terms of the Creative Commons Attribution 3.0 License. Further distribution of this work must maintain attribution to the author(s) and the published article's title, journal citation, and DOI. particle beam loses and deposits energy mostly in the form of heat in the intercepting device or it is scattered away from the nominal orbit.

At CERN, energies of the accelerated particle beams range from a few $\mathrm{KeV}$ to $7 \mathrm{TeV}$ and within time intervals ranging from $n s$ to $m s$ resulting in high temperature gradients which in turn give rise to severe quasistatic or dynamic stresses [6-8]. Temperatures may rise to the point of phase change [8] although in many cases where phase change is not present, temperatures can still rise enough to cause structural failure [9]. Stresses are a direct consequence of the induced temperature field that can also result in failure.

Analytical studies for rapid heating and the associated thermoelastic problem using lasers or welding processes have been extensively studied in [10-13] for Gaussian distribution sources where even nonlinearities are taken into account. An analytical solution for a moving laser pulse modeled as a moving Gaussian is given by [14]. Dynamic thermoelasticity in a finite space using a uniformly distributed source is well explained in [15] and is simple to obtain from Sturm-Liouville theory. In the finite space problem one must be careful when truncating the infinite series solutions as convergence maybe dependent on the parameters of the problem, for example velocity of sound or size of the Gaussian source. 
The need for three dimensional solutions for the infinite space thermoelastic problem has grown with the development of accelerator technology. Analytical solutions of the nondimensionalized problem in infinite space are very useful for the initial stages of the design of a BID. Such analytical solutions, which are absent from the literature, offer a physical insight into phenomena not revealed by numerical simulations.

In the process of designing a BID one needs to know whether or not dynamic effects should be taken into account. Dynamic effects are often dominant in the time frame of a repetition, that is for time intervals of the order of one to several pulse lengths. Solving for dynamic elasticity is usually achieved by finite elements software as the geometries and boundary conditions can be complex [16]. In order to capture the dynamic effects and with such small particle beam widths and high velocities of sound, CFL conditions render these computations very lengthy $[6,17]$. In many cases the size of the BID is large enough so that any reflections from the boundaries can be neglected for time intervals of the order of a pulse. The assumption of infinite geometries comes naturally and its extent and limitations are shown herein.

The purpose of this article is to shed light on the specific mechanisms of thermal stresses when a solid undergoes a thermal shock with Gaussian profile in space during the time interval of a pulse length. Stress fields are given analytically using well-known functions. Parametric studies which can dramatically reduce the design process time with respect to beam parameters and material properties are therefore foreseeable. The expressions developed herein can also be used to compare the dynamic effects versus the quasistatic approximation and allow us to decide whether a dynamic computation is necessary.

This analysis is applicable not only for the design of BID's but also for proton beam cancer therapy [18-20]. In the latter proton beams are used to kill cancer cells in while minimizing radiation in healthy areas around the cancer cells. However it appears that the issue of possible collateral damages due to the stress field in the brain has not yet been studied in the literature. Linear thermoelasticity represents a rather bold assumption for such viscoelastic materials but it gives an idea of the order of magnitude of the expected stresses.

The article is structured in the following way. The basic equations of uncoupled thermoelasticity, also called theory of thermal stresses, are laid down and all necessary assumptions are presented. The problem is then nondimensionalized and solutions to the potential equations are sought using Green's functions, Eqs. (14) and (17). Von Mises stresses are derived from the elastic potential for both the quasistatic and general solutions, Eqs. (22) and (24). The validity of matching the infinite medium with a finite geometry is sought and a parametric analysis on the error of the results is given. Furthermore under a valid infinite medium assumption, the quasistatic solution is compared to its dynamic counterpart with respect to nondimensional parameters. Finally a case study is presented where all the analytical results are applied and compared to bounded medium solutions. The bounded medium solutions are in the form of infinite series for which a convergence evaluation is given in order to control the truncated series error.

\begin{tabular}{lc} 
& Nomenclature \\
$\alpha$ & thermal expansion coefficient \\
$\lambda$ & Lamé first parameter \\
$\mu$ & Lamé second parameter \\
$\theta$ & temperature \\
$\rho$ & density \\
$k$ & thermal conductivity \\
$c$ & specific heat \\
$c_{0}$ & longitudinal wave velocity \\
$A$ & peak of power density function \\
$t_{p}$ & pulselength \\
$\sigma$ & standard deviation of Gaussian power density \\
$\Pi_{1}$ & non-dimensional thermal diffusivity \\
$\Pi_{2}$ & non-dimensional standard deviation of Gaussian power \\
\multicolumn{1}{c}{ density } \\
$\Pi_{3}$ & non-dimensional thermal expansion \\
$\Pi_{4}$ & non-dimensional longitudinal wave velocity \\
$\Pi_{5}$ & non-dimensional parameter \\
$\Pi_{6}$ & non-dimensional shear wave velocity \\
$\Pi_{c}$ & peak of non-dimensional power density function \\
$E_{\mathrm{err}}$ & relative error between dynamic and static displacement \\
& averages \\
$\epsilon_{\mathrm{rms}}$ & relative error of the partial sums \\
\hline &
\end{tabular}

\section{PROBLEM DESCRIPTION}

Particle beam intercepting devices are dedicated to absorb large amounts of energy in relatively short time intervals. Thermally induced stresses pose a major threat to the BID's and their computation is somewhat cumbersome. The equations of linear thermoelasticity presented in [21] are usually solved by means of lengthy numerical methods (finite elements) and can cripple the time constraint of a design process when considering such thermal stresses.

We will confine ourselves to the study of thermoelastic displacements in infinite media. This simplifies the problem such that the shear potential of the uncoupled equations of thermoelasticity is automatically reduced to zero. The beam as well as the heat deposition profile near the Bragg peak are assumed to be Gaussian. By consequence the heat source in the heat diffusion equation is assumed to be a spherically perfect Gaussian. In addition, no heat diffusion is assumed within the time interval of a pulse. The nondiffusive assumption can be easily verified by using the solutions developed in [22]. The case study herein is treated with the adiabatic assumption and the error of the real temperature fields is given. The temperature field is therefore directly proportional to the Gaussian heat source. Solutions to the longitudinal wave potential equation with a 
Gaussian source term are sought. The influence of the inertia terms is analyzed by comparing solutions of the quasistatic problem to the ones obtained from the longitudinal wave equation. Finally the proposed solution for the assumed infinite medium is compared to the series expansion solution for a finite medium.

\section{ANALYTICAL SOLUTIONS OF THE ELASTIC POTENTIAL FOR INFINITE MEDIA}

The classical uncoupled equations of thermoelasticity with no displacement dependent term in the heat equation are [21],

$$
\begin{gathered}
(\lambda+\mu) \nabla(\nabla \cdot \mathbf{u})+\mu \nabla^{2} \mathbf{u}-\alpha(3 \lambda+2 \mu) \nabla\left(T-T_{0}\right) \\
=\rho \frac{\partial^{2} \mathbf{u}}{\partial t^{2}} \\
k \nabla^{2} T=\rho c_{v} \frac{\partial T}{\partial t}-g
\end{gathered}
$$

where the constants $\lambda, \mu, \rho, \alpha, c_{v}$, and $k$ are respectively the Lamé parameters, density, coefficient of thermal expansion, specific heat and coefficient of thermal conductivity. The vector $\mathbf{u}$ and the scalar $T$ are respectively the displacement field and the temperature field and are both functions of space and time. $T_{0}$ represents the initial temperature field in spherical coordinates $T(r, 0)$ which will be assumed to be constant. Finally $g(r)=A \exp \left(-r^{2} / 2 \sigma^{2}\right)$ represents the heat source term, with $\sigma$ being equal to the geometric average of the three standard deviations of a spatial Gaussian fit to the power deposition map.

The heat equation contains no displacement dependent terms so that we can solve for the temperature $T(r, t)$ in an infinite medium where a decay at infinity is assumed and a constant initial temperature field. The difference between the temperature and the initial temperature appears in the temperature dependent term of the wave equation so that we set $\theta(r, t)=T(r, t)-T_{0}$ and solve for $\theta(r, t)$. Assuming no heat diffusion during the short interval of time the solution for $\theta(r, t)$ becomes trivial to yield,

$$
\theta(r, t)=\frac{g(r)}{\rho c_{v}} t .
$$

The errors in the approximation of zero diffusivity are treated in [22], where ratios between the theoretical and zero diffusivity dimensionless peak temperatures are given. The case study presented in this paper yields a ratio very close to 1 , which is also seen in many other BID applications. The displacement field has homogeneous initial conditions such that $\mathbf{u}(r, 0)=0$ and $\partial \mathbf{u} /\left.\partial t\right|_{t=0}=0$. Following the classical way to decouple Eq. (1) by taking the divergence and the curl we end up with two potential equations, one for the longitudinal displacement and one for the shear in terms of their respective potentials $\Phi(r, t)$ and $\boldsymbol{\Psi}(r, t)$ such that $\mathbf{u}(r, t)=\nabla \Phi+\nabla \wedge \boldsymbol{\Psi}$. The solution for the latter is trivial due to the homogeneous initial conditions and the source term acting only on the longitudinal expansion of the material. There is only one equation to solve for the longitudinal waves, expressed in terms of $\Phi(r, t)$ that reads,

$$
(\lambda+2 \mu) \nabla^{2} \Phi=\rho \frac{\partial^{2} \Phi}{\partial t^{2}}+\alpha(3 \lambda+2 \mu) \theta,
$$

with homogeneous initial conditions. After rearranging terms and showing the source term explicitly we obtain,

$$
\nabla^{2} \Phi=\frac{1}{c_{0}^{2}} \frac{\partial^{2} \Phi}{\partial t^{2}}+B t \exp \left(\frac{-r^{2}}{2 \sigma^{2}}\right)
$$

where $c_{0}=\sqrt{\frac{\lambda+2 \mu}{\rho}}$ stands for the speed of the longitudinal waves and $B=\frac{\alpha(3 \lambda+2 \mu)}{(\lambda+2 \mu)} \frac{A}{\rho c_{v}}$.

\section{A. Nondimensionalization of the problem}

Let $\sigma$ be the characteristic length scale of the domain and $t_{p}$ be the reference time representing the time of the pulse. The following new variables are obtained,

$$
r^{*}=\frac{r}{\sigma}, \quad t^{*}=\frac{t}{t_{p}}, \quad \text { and } \quad \Phi^{*}=\frac{\Phi}{\sigma^{2}} .
$$

Inserting the above variables into Eq. (5) and dropping the star notation from now on, the following dimensionless equation is obtained,

$$
\nabla^{2} \Phi=\frac{1}{\Pi_{4}^{2}} \frac{\partial^{2} \Phi}{\partial t^{2}}+\Pi_{c} t \exp \left(\frac{-r^{2}}{2}\right)
$$

where $\Pi_{c}=\Pi_{3} \Pi_{5}$ and $\Pi_{2}, \Pi_{3}, \Pi_{4}, \Pi_{5}$, and $\Pi_{6}$ are five independent dimensionless parameters that read,

$$
\begin{aligned}
& \Pi_{2}=\frac{L}{\sigma}, \\
& \Pi_{3}=\frac{\alpha A t_{p}}{\rho c}, \\
& \Pi_{4}=\frac{c_{0} t_{p}}{\sigma}, \\
& \Pi_{5}=\frac{(3 \lambda+2 \mu)}{\lambda+2 \mu}, \\
& \Pi_{6}=\sqrt{\frac{\lambda}{\rho} \frac{t_{p}}{\sigma},}
\end{aligned}
$$

where the quantity defined as $\sqrt{\lambda / \rho}$ is used instead of the shear velocity due to simplifications that arise in the stress calculations. The same dimensionless numbers can be obtained using the П-Buckingham theorem. The characteristic length $L$ is used to provide comparison of the infinite medium solutions with their finite medium counterparts by comparing their behavior in the normalized sphere $r \leq \Pi_{2}$. 


\section{B. Quasistatic solutions of the elastic potential}

When displacements change slowly with time, inertia terms can be assumed negligible to yield a quasistatic ordinary differential equation which is,

$$
\frac{1}{r} \frac{d^{2}\left(r \Phi_{Q}\right)}{d r^{2}}=\Pi_{c} t \exp \left(\frac{-r^{2}}{2}\right)
$$

where time only appears in the source term and the subscript $Q$ stands for quasistatic. We will refer to this problem in the forthcoming sections as the "quasistatic assumption." The solution is given by,

$$
\Phi_{Q}(r, t)=-\frac{\sqrt{2 \pi} \Pi_{c}}{2} \frac{t}{r} \operatorname{erf}\left(\frac{r}{\sqrt{2}}\right) .
$$

\section{General solutions of the elastic potential}

In the spherical problem where the source is a spherical Gaussian function we expect the solution to be independent of the polar and azimuthal angles. By setting $\Psi(r, t)=$ $r \Phi(r, t)$ in Eq. (5) we obtain an equation for $\Psi$ with the Laplacian in one-dimensional Cartesian coordinates and $r \geq 0$. Using the method of images [23] we construct the Green's function for this problem that reads,

$$
\begin{aligned}
G(R, \hat{R}, \tau) & =G_{1}(R, \tau)-G_{2}(\hat{R}, \tau) \\
& =-H(\tau) \frac{\Pi_{4}}{2}\left(H\left(\tau-\frac{R}{\Pi_{4}}\right)-H\left(\tau-\frac{\hat{R}}{\Pi_{4}}\right)\right),
\end{aligned}
$$

where $R=r-r^{\prime}, \hat{R}=r+r^{\prime}, \tau=t-t^{\prime}$ and $r^{\prime}, t^{\prime}$ being dummy variables. The solution for $\Psi$ is given by [23]; considering homogeneous initial conditions $\Psi$ reads,

$$
\begin{aligned}
\Psi(r, t)= & \int_{0}^{t} \int_{0}^{\infty} G_{1} \Pi_{c} t^{\prime} r^{\prime} \exp \left(\frac{-r^{\prime 2}}{2}\right) d r^{\prime} d t^{\prime} \\
& -\int_{0}^{t} \int_{0}^{\infty} G_{2} \Pi_{c} t^{\prime} r^{\prime} \exp \left(\frac{-r^{\prime 2}}{2}\right) d r^{\prime} d t^{\prime}
\end{aligned}
$$

and $\Phi$ is given by,

$$
\begin{aligned}
\Phi(r, t) & =\frac{-\Pi_{c}}{4 \Pi_{4}} \frac{1}{r} \cdot\left[2 \left(\exp \left(\frac{-\left(r-\Pi_{4} t\right)^{2}}{2}\right)\right.\right. \\
& \left.-\exp \left(\frac{-\left(r+\Pi_{4} t\right)^{2}}{2}\right)\right) \\
& +\sqrt{2 \pi}\left(-\left(r-\Pi_{4} t\right)\left(\operatorname{erf}\left(\frac{r}{\sqrt{2}}\right)-\operatorname{erf}\left(\frac{r-\Pi_{4} t}{\sqrt{2}}\right)\right)\right. \\
& \left.\left.+\left(r+\Pi_{4} t\right)\left(\operatorname{erf}\left(\frac{r}{\sqrt{2}}\right)-\operatorname{erf}\left(\frac{r+\Pi_{4} t}{\sqrt{2}}\right)\right)\right)\right] \cdot
\end{aligned}
$$

We will refer to this solution as the "general problem" solution.

\section{ANALYTICAL EXPRESSION OF THE VON MISES STRESS FOR INFINITE MEDIA}

\section{A. The Von Mises stress criterion}

Different definitions of equivalent stresses apply for different materials [24]. Given many beam dump materials are isotropic and ductile, the Von Mises equivalent stress is most adequate for the purpose of our study and the spherical symmetry of the problem allows one to derive it from the elastic potential. Since $\sigma_{\mu \mu}=\sigma_{\nu \nu}$, the Von Mises stress simplifies to: $\sigma_{\mathrm{VM}}=\left|\sigma_{r r}-\sigma_{\mu \mu}\right|$ where $\sigma_{r r}$ and $\sigma_{\mu \mu}$ are given in Eqs. (18) and (19) as,

$$
\begin{aligned}
& \sigma_{r r}=\Pi_{4}^{2} \frac{\partial^{2} \Phi}{\partial r^{2}}+\Pi_{6}^{2} \frac{2}{r} \frac{\partial \Phi}{\partial r} \\
& \sigma_{\mu \mu}=\sigma_{\nu \nu}=\Pi_{6}^{2} \frac{\partial^{2} \Phi}{\partial r^{2}}+\frac{\Pi_{4}^{2}+\Pi_{6}^{2}}{r} \frac{\partial \Phi}{\partial r} .
\end{aligned}
$$

Thus the Von Mises Criteria relation to the elastic potential reads:

$$
\sigma_{\mathrm{VM}}=\left|\Pi_{4}^{2}-\Pi_{6}^{2}\right|\left|\frac{\partial^{2} \Phi}{\partial r^{2}}-\frac{1}{r} \frac{\partial \Phi}{\partial r}\right| .
$$

Of particular interest are the localization and maximum values of the underlying equivalent stresses attained within $0 \leq r \leq \Pi_{2}$.

\section{B. Localization and value of extrema of the quasistatic solution}

The solution of the quasistatic elastic potential is given in (14) and the expressions of the Von Mises equivalent stress for the quasistatic solution is derived by inserting Eq. (14) in (20) to obtain the following dimensionless expression,

$$
\begin{aligned}
\sigma_{\mathrm{VM}}^{Q}= & \left|\Pi_{4}^{2}-\Pi_{6}^{2}\right|\left|\frac{\sqrt{2 \pi} \Pi_{c}}{2} t\right| \\
& \cdot\left|\frac{3 \operatorname{erf}\left(\frac{r}{\sqrt{2}}\right)-\sqrt{\frac{2}{\pi}} r\left(3+r^{2}\right) \exp \left(-\frac{r^{2}}{2}\right)}{r^{3}}\right| .
\end{aligned}
$$

From this equation it is clear that $\sigma_{\mathrm{VM}}^{Q}$ evolves linearly with time so that the position of the peak of stresses necessarily occurs at the end of the pulse. In addition it is also clear that the radius $r_{\max }$ where the peak of stress occurs for a given time is the same for all times. By solving this equation numerically using MATLAB [25] we get $r_{\max } \simeq 1.775$. By inserting $r_{\max }=1.775$ in Eq. (21) the maximum of the now dimensional Von Mises equivalent stress in the quasistatic assumption reads:

$$
\sigma_{\mathrm{VM}}^{Q^{\max }}=2 \mu \cdot \Pi_{c} \cdot \zeta_{q}
$$




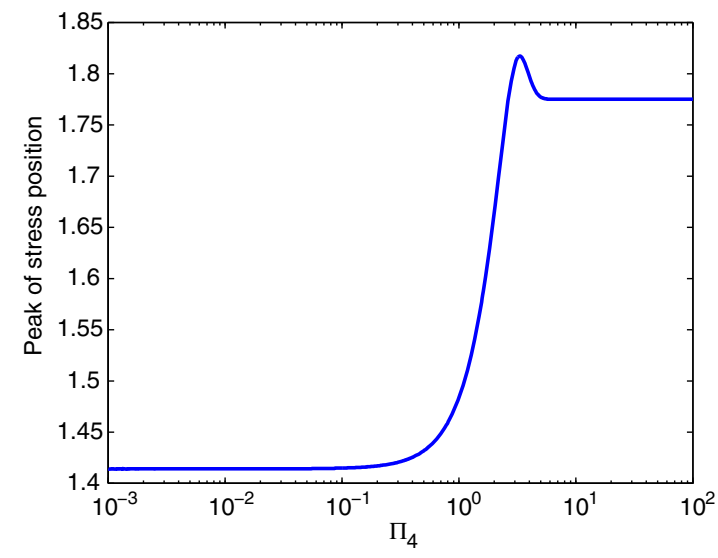

FIG. 1. Position of the peak of stress for the general problem. For $\Pi_{4}$ sufficiently large the peak position matches the quasistatic result.

where $\zeta_{q}$ is a constant approximately equal to 0.2173 and $\mu$ is the shear modulus of the material.

\section{Localization and value of extrema of the general solution}

In the general thermoelastic problem, the elastic potential given by Eq. (17), is inserted in Eq. (20) to obtain the dimensionless Von Mises stress,

$$
\begin{aligned}
\sigma_{\mathrm{VM}}= & \left|\Pi_{4}^{2}-\Pi_{6}^{2}\right|\left|\Pi_{c}\right| \\
& \cdot\left|\frac{d^{2} \hat{\Phi}\left(r, t, \Pi_{4}\right)}{d r^{2}}-\frac{\frac{d \hat{\Phi}\left(r, t, \Pi_{4}\right)}{d r}}{r}\right|,
\end{aligned}
$$

where $\hat{\Phi}=\frac{1}{\Pi_{c}} \Phi$. A graph of this function is presented on Fig. 8 in the case study section. Note that the stress is zero at the center due to the point symmetry of the problem.

Equation (23) shows that the localization of the maximum a priori depends on two variables, namely: $t$ and $\Pi_{4}$. The linearity of the material parameters, the constant power source and the absence of any reflective boundaries suggest the peak of stresses will occur at the end of the pulse, confirmed by the positive sign of the derivative of stress with respect to time. The localization of the maximum dependence reduces to $\Pi_{4}$.

The position of the peak of stress for all $\Pi_{4}$ in the range $\left[10^{-3}, 10^{2}\right]$ is sought using MATLAB [25] and presented on Fig. 1. Two regions stand out. The first region is at approximately $r=1.414$ where dynamic effects are dominant and the second near $r=1.775$ for $\Pi_{4}$ sufficiently large which correspond to the quasistatic case.

The maximum dimensional Von Mises stress in the general problem reads,

$$
\sigma_{\mathrm{VM}}^{\max }=2 \mu \cdot \Pi_{c} \cdot \zeta\left(\Pi_{4}\right),
$$

where $\zeta$ is a function of $\Pi_{4}$ shown on Fig. 2 and 3.

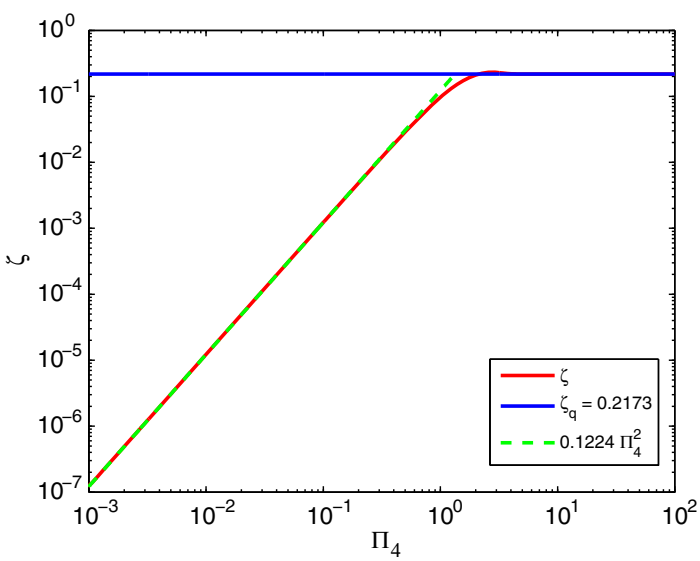

FIG. 2. $\zeta$ function as presented in Eq. (23) and its asymptotes. Since $\zeta$ is increasing with $\Pi_{4}$ nearly everywhere, so is the Von Mises stress. The asymptote for high values of $\Pi_{4}$ matches $\zeta_{q}$ as expected.

\section{Comparison of the quasistatic and general VM stresses}

The $\zeta$ function appearing in the equation of the general Von Mises (VM) stress [Eq. (24)] admits two asymptotes. The asymptote for sufficiently large values of $\Pi_{4}$ corresponds to the quasistatic case where $\zeta\left(\Pi_{4}\right) \simeq \zeta_{q}$. The asymptote for sufficiently small values of $\Pi_{4}$ has the equation: $\zeta\left(\Pi_{4}\right) \simeq 0.1224 \cdot \Pi_{4}^{2}$. In the transition region, no approximation is available and one needs to rely on Fig. 3 to calculate the Von Mises stress.

From Fig. 2 we conclude that the VM stress in the quasistatic assumption is always higher than in the general problem. Therefore Eq. (22) provides a supremum to the maximum of the Von Mises stress in the general problem as long as the quasistatic stress value is below the proportional limit. The two solutions are virtually the same for values of $\Pi_{4}>4$.

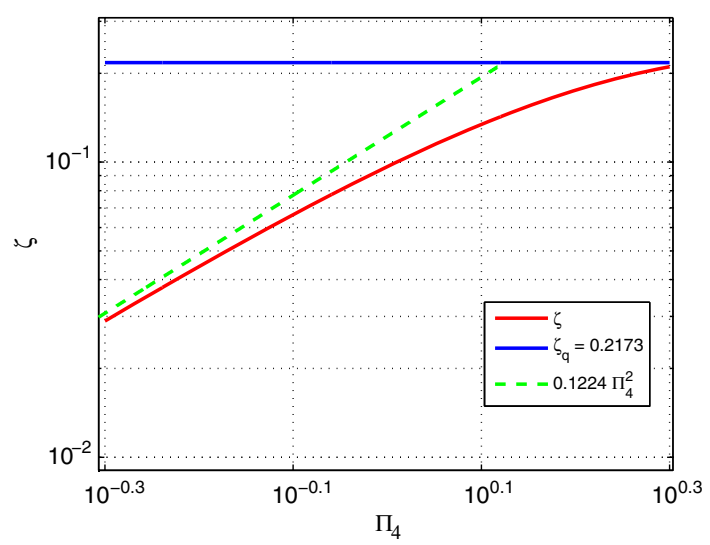

FIG. 3. $\zeta$ function as presented in Eq. (23) in the region where it cannot be approximated by one of its asymptote with less than $10 \%$ of error. 


\section{E. Optimization algorithm for maximum principal stresses at time $t$}

Unless maximum values are reached on the boundaries of the domain i.e. $r=\Pi_{2}$, they will occur within the domain of $0 \leq r \leq \Pi_{2}$ where the first derivatives of the optimized quantities are zero. Solving exactly for $\partial \sigma_{r r} / \partial r=0$ and $\partial \sigma_{\nu \nu} / \partial r=\partial \sigma_{\mu \mu} / \partial r=0$ or a Von Mises criterion, can be cumbersome so we propose an algorithm for evaluating the maximum stress location $r_{\max }$. Let

$$
S=a \frac{\partial^{2} \Phi}{\partial r^{2}}+\frac{b}{r} \frac{\partial \Phi}{\partial r}
$$

with $\Phi=\Psi / r$ to yield the general form of,

$$
S=\frac{N(r)}{r^{3}},
$$

where $\quad N(r)=a \Psi^{\prime \prime} r^{2}+(-2 a+b) \Psi^{\prime} r+(2 a-b) \Psi$, with prime denoting differentiation with respect to $r$. Taking,

$$
\frac{\partial S}{\partial r}=\frac{r N^{\prime}-3 N}{r^{4}}=0,
$$

solves for the critical point noted $r_{c}$. Now consider the class of functions $\hat{N}(r)$ that solves the differential equation: $r \hat{N}^{\prime}-3 \hat{N}=0$ on $r \in\left[0, \Pi_{2}\right]$. These functions are the functions: $\hat{N}(r)=C r^{3}$ with $C$ an arbitrary constant. Since $\mathrm{C}$ is any real constant, there is one of them, denoted by $C_{c}$, such that:

$$
N\left(r_{c}\right)=C_{c} r_{c}^{3} .
$$

Let $\hat{N}_{c}(r)=C_{c} r^{3}$. It is clear that $N(r)$ and $\hat{N}_{c}(r)$ cross at $r_{c}$. Let us prove that $N(r)$ and $\hat{N}_{c}(r)$ are also tangent at $r_{c}$, i.e. let us prove that $N^{\prime}\left(r_{c}\right)=\hat{N}_{c}{ }^{\prime}\left(r_{c}\right)$. From Eq. (27), $N^{\prime}\left(r_{c}\right)=3 N\left(r_{c}\right) / r_{c}$ and by definition of $C_{c}$ and $\hat{N}(r)$ : $3 N\left(r_{c}\right) / r_{c}=3 \hat{N}\left(r_{c}\right) / r_{c}=3 C_{c} r_{c}^{2}$. Since $\hat{N}(r)$ is a cubic function, $3 C_{c} r_{c}^{2}=\hat{N}_{c}^{\prime}\left(r_{c}\right)$.

To conclude, the peak of the $S$ function can be found by finding the cubic function: $\mathrm{Cr}^{3}$ that intersects and is tangent to the $N$ function easily derived from $S$. The point of intersection is the critical radius. For the sake of illustration we set $\Pi_{2}=19.9$ and $\Pi_{4}=1.64 \times 10^{-3}$ which are the values used in the case study in Sec. VII. For the Von Mises criterion $a=-b=1$ and $N(r)$ follows form the equation stated above. In Fig. 4(b) is a plot of $N(r)$ together with a plot of $\hat{N}(r)=\mathrm{Cr}^{3}$ where the tangential intersection is for a specific value of $C=2.1622 \times 10^{-9}$. For a given sweep precision of the coefficient $C$ the intersection and therefore the extremum point of the Von Mises stress is at
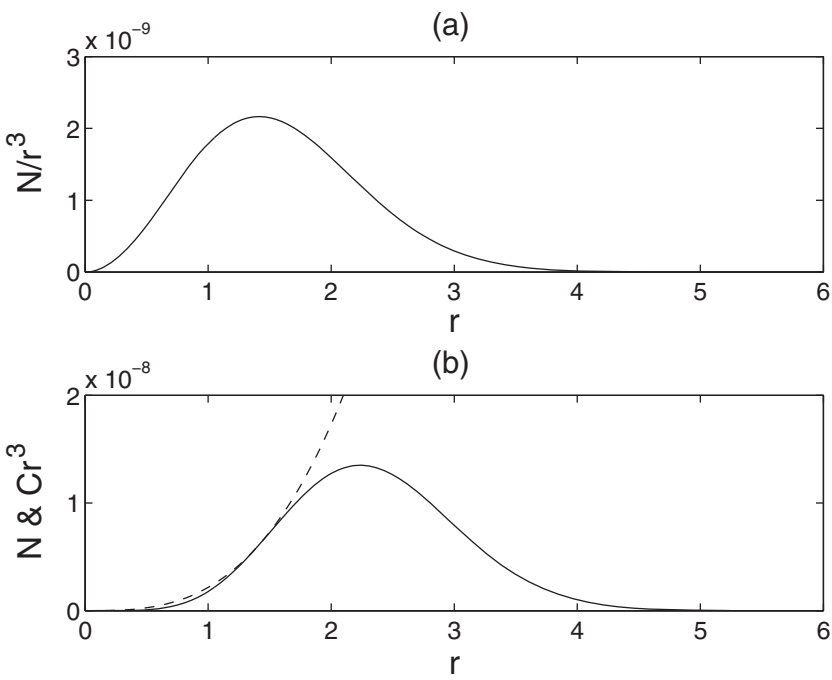

FIG. 4. In panel (a) the Von Mises stresses $\left(N(r) / r^{3}\right)$ are in solid where the extremum is aligned with the intersection of the two curves in panel (b) containing the plots of $N(r)$ in solid and $\hat{N}(r)=C r^{3}$ in dashed.

$1.3874<r<1.4421$ with a relative width of the ordinate interval of 0.1227 .

\section{MATCHING THE INFINITE MEDIUM SOLUTIONS ONTO A FINITE SPHERE}

Equations (14) and (17) are exact for infinite media. If one considers a finite medium these solutions can be used provided the above dimensionless parameters fall within determined intervals. As a reminder, the heat source is a perfect spherical Gaussian which acts on the body during a very short time $t_{p}$ and as a consequence heat diffusion can be neglected. An estimate of the error for such an assumption is given in [22], where parameters $\Pi_{1}=$ $D t_{p} / L$ and $\Pi_{2}$ need to fall within some interval for a specified error, where $D$ represents the thermal diffusivity. It is therefore important to validate the assumption of nondiffusion before using a solution presented herein.

\section{A. Validity of the general solution-The ideal region}

The relevance of our model depends on the intervals of validity in space and time of Eq. (17). The general solution is not always exact for all pulse lengths and length scales of the intercepting device.

Let us consider a perfectly spherical ball of radius $L$ with the Gaussian source impacting at its center. We define $L$ as the distance separating the peak of energy deposition to the closest surface of the BID.

At time $t=0$, the infinite domain solution and the finite solution given by Eqs. (17) and (35) respectively are identical over the whole ball. Because of propagating waves, at time $t=\epsilon$ where $\epsilon \ll 1$, the region where the two solutions are identical has shrunk. The key question is 
thus to describe the evolution with time of the region where the two solutions remain identical.

At time $t=\epsilon$ the Gaussian source will give some amount of energy to all points of the ball. A first wave front, created by the reflection of the boundary of the system, will propagate inward. This wave front, not taken into account in the infinite solution, propagates at sound speed toward the center of the ball. The finite and infinite solutions are identical in the region that has not yet seen this wave front.

If the normalized domain is a sphere or radius $\Pi_{2}=L / \sigma$, $I(t)$ is the ideal region, and $B(c, r)$ is a ball of center $c$ and radius $r$, then:

$$
I\left(t_{p}\right)= \begin{cases}B\left(0, \Pi_{2}-\Pi_{4}\right) & \text { if } \Pi_{2}>\Pi_{4} \\ 0 & \text { otherwise }\end{cases}
$$

Therefore if the wave front has not had the time to reach the radius at which the peak of stress occurs, i.e. if $r_{\max }<\Pi_{2}-\Pi_{4}$, Eqs. (22) and (24) still hold.

\section{B. Validity of quasistatic elasticity}

Another time saving assumption is that of quasistatic thermoelasticity. Modeling a finite medium with quasistatic elasticity in infinite media raises an important issue, that of quasistatic elasticity and nondiffusive heat deposition. The error caused by the assumption of nondiffusive energy deposition is evaluated from [22]. For a fast heat source term to yield a slow varying stress field it is necessary to define a new error which depends on $\Pi_{4}$ and represents a normalized relative error of the two displacements averages reading,

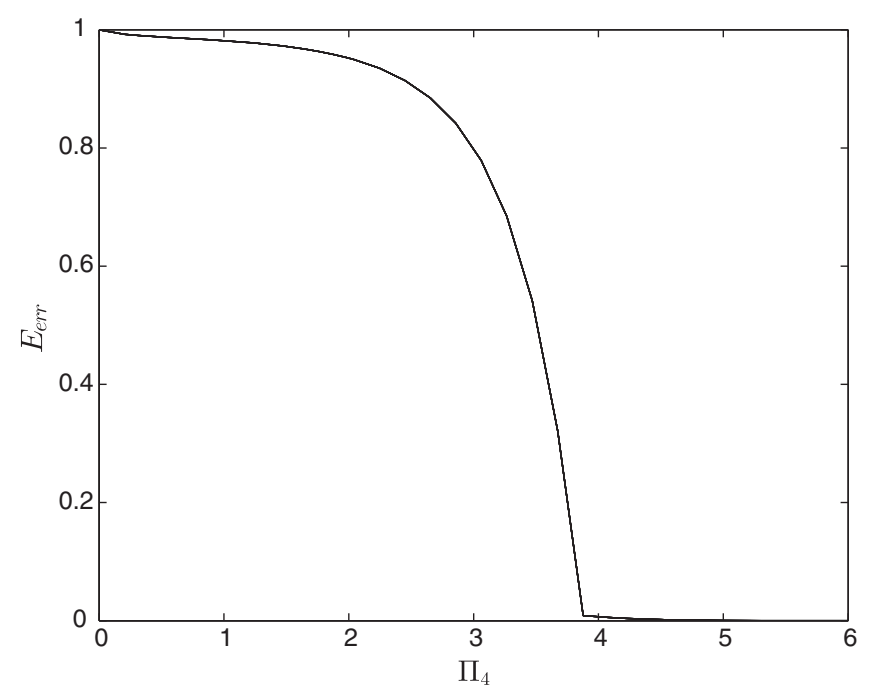

FIG. 5. Plot of $E_{\text {err }}$ versus $\Pi_{4}$ showing the value of $\Pi_{4} \sim 3.88$ at which the general solution becomes indistinguishable from the quasistatic one.

$$
E_{\mathrm{err}}=\left|\frac{\sigma_{\mathrm{VM}}^{\max }-\sigma_{\mathrm{VM}}^{Q^{\max }}}{\sigma_{\mathrm{VM}}^{\max }}\right| .
$$

A contour plot of $E_{\text {err }}$ versus $\Pi_{4}$ is presented in Fig. 5. Coefficients of $E_{\text {err }}$ near zero show that dynamical effects are indistinguishable from the quasistatic solution. Values near one imply the opposite. By combining the information in Fig. 5 and also verifying the near zero diffusion assumption the infinite medium equations can be used rigorously.

\section{SERIES SOLUTIONS}

Infinite series solutions are here compared to the solution obtained by Eq. (17). We wish to point out that they do not represent a novelty and will be used solely for comparison purposes. Such solutions are well known and can be derived using Sturm-Liouville theory [26]. The geometry consists of a sphere of normalized radius equal to $\Pi_{2}$. Solutions are sought for $0 \leq r \leq \Pi_{2}$. Clamped or traction free boundary conditions and homogeneous initial conditions are taken into account. Note that we do not consider shear waves since the source term is radial and no shear waves will emerge from the coupling of the compressive and shear fields at the boundaries.

\section{A. Bounded dynamic elasticity}

The solution to Eq. (7) is of the form,

$$
\Phi_{S}(r, t)=\sum_{n=0}^{\infty} A_{n}(t) \phi_{n}(r)
$$

where coefficients $A_{n}(t)$ are retrieved by inserting the previous expression into Eq. (7) to obtain,

$$
\frac{d^{2} A_{0}}{d t^{2}}+\sum_{n=1}^{\infty}\left(\frac{d^{2} A_{n}}{d t^{2}}+\beta_{n}^{2} A_{n}\right) \phi_{n}=-f(r, t),
$$

where $\beta_{n}^{2}=\Pi_{4}^{2} \lambda_{n}^{2}$ and $f(r, t)=\Pi_{4}^{2} \Pi_{c} t \exp \left(-r^{2} / 2\right)$ and respective solutions,

$$
\begin{aligned}
A_{n}(t)= & C_{n} \cos \left(\beta_{n} t\right)+D_{n} \sin \left(\beta_{n} t\right)+K_{n}(t) \frac{1}{\beta_{n}^{2}}, \\
& \text { for } n \in \mathbb{Z}^{+}, \quad \text { and } \\
A_{0}(t)= & \frac{K_{0}(t)}{6} t^{2}+E_{0} t+D_{0},
\end{aligned}
$$

where $K_{n}(t)=-\frac{\int_{0}^{\Pi_{2}} f(r, t) \phi_{n}(r) w d r}{\int_{0}^{\Pi_{2}} \phi_{n}(r)^{2} w d r}$ and $K_{0}(t)=-\frac{\int_{0}^{\Pi_{2}} f(r, t) w d r}{\int_{0}^{\Pi_{2}} w d r}$. $w=r^{2}$ is a weight function that depends on the coordinate system and is necessary to satisfy orthogonality of the eigenfunctions $\phi_{n}$. The eigenvalues are shown as $\lambda_{n}$ which brings us to solve the eigenvalue problem in order to obtain the $\phi_{n}$. The associated eigenproblem reads, 


$$
\nabla^{2} \phi_{n}+\lambda_{n}^{2} \phi=0
$$

and is solved for $u\left(\Pi_{2}, t\right)=0$ where $\mathbf{u}=\nabla \Phi_{S}$.

\section{Clamped boundary conditions}

The eigenfunctions of Eq. (34) are spherical Bessel functions noted $\phi_{n}(r)=j_{0}\left(\lambda_{n} r\right)$ and the associated eigenvalues are derived using the Dirichlet condition on the displacement or $d \phi_{n} /\left.d r\right|_{r=\Pi_{2}}=0$ that implies $\lambda_{n} j_{1}\left(\lambda_{n} \Pi_{2}\right)=0$. Zero is an obvious eigenvalue with $\phi_{0}=1$ as its associated eigenfunction. Inserting the eigenfunctions back into Eq. (31) and imposing the homogeneous initial conditions on the displacement $[u(r, 0)=0$ and $\dot{u}(r, 0)=0$ ] we obtain,

$\Phi_{S}(r, t)=\frac{K_{0}(t)}{6} t^{2}+\sum_{n=1}^{\infty} \frac{K_{n}(t)}{\beta_{n}^{2}}\left(1-\frac{\sin \left(\beta_{n} t\right)}{\beta_{n} t}\right) \phi_{n}(r)$,

where $K_{0}$ and $K_{n}$ are,

$K_{0}(t)=\frac{3 \Pi_{c} \Pi_{4}^{2} t}{\Pi_{2}^{3}}\left[\Pi_{2} \exp \left(\frac{-\Pi_{2}^{2}}{2}\right)-\frac{\sqrt{\pi}}{2} \operatorname{erf}\left(\frac{\Pi_{2}}{\sqrt{2}}\right)\right]$

and

$$
\begin{aligned}
K_{n}(t)= & \frac{2 \lambda_{n} \Pi_{c} \Pi_{4}^{2} t}{\Pi_{2} \sin \left(\lambda_{n} \Pi_{2}\right)^{2}} \\
& \times\left\{\exp \left(\frac{-\Pi_{2}^{2}}{2}\right) \sin \left(\lambda_{n} \Pi_{2}\right)+-\frac{\sqrt{2 \pi}}{4} \lambda_{n} \exp \left(\frac{-\lambda_{n}^{2}}{2}\right)\right. \\
& \left.\times\left[\operatorname{erf}\left(\frac{\Pi_{2}-i \lambda_{n}}{\sqrt{2}}\right)+\operatorname{erf}\left(\frac{\Pi_{2}+i \lambda_{n}}{\sqrt{2}}\right)\right]\right\}
\end{aligned}
$$

Traction free boundary conditions can also be considered to yield a solution of the form of Eq. (35). The eigenvalues $\lambda_{n}$ 's take different values from applying traction free boundary conditions on the surface which read,

$$
\left.\sigma_{r r}\right|_{r=\Pi_{2}}=\left.\left(\Pi_{4}^{2} \frac{\partial^{2} \Phi_{S}}{\partial r^{2}}+\Pi_{6}^{2} \frac{2}{r} \frac{\partial \Phi_{S}}{\partial r}\right)\right|_{r=\Pi_{2}}=0
$$

We do not expand the traction free solution any further as it is not in the scope of the present study.

\section{Relative error of the partial sums}

The series solutions will be used for comparison purposes. It is therefore necessary to know the error induced by truncating the series at the $m^{\text {th }}$ term. Equation (32) is of the form $\sum_{n=0}^{\infty} K_{n}(t) \phi_{n}(r)=-f(r, t)$. Using the orthogonality of the eigenfunctions the relative root mean square (rms) of the difference between dimensionless temperature $f(r, t)$ and the partial sum $S_{m}=\sum_{n=0}^{m} K_{n}(t) \phi_{n}(r)$, is,

$$
\epsilon_{\mathrm{rms}}\left(\Pi_{2}, m\right)=\sqrt{\frac{\int_{V} f^{2} d V-\sum_{n=0}^{m} K_{n}^{2} \int_{V} \phi_{n}^{2} d V}{\int_{V} f^{2} d V}}
$$

\section{APPLICATIONS}

In order to illustrate the above models we propose a case study which concerns the PSB dump at CERN [4]. The proton beam is extracted from the PSB at energies of the order of $2 \mathrm{GeV}$ and is intercepted by a beam dump. The temperature rise after one pulse is of the order of $150 \mathrm{~K}$ and the stresses are of the order of $100 \mathrm{MPa}$ of which $60 \mathrm{MPa}$ are due to dynamic effects [4]. Results in [4] will be used to validate the analytical model.

The quasistatic study failed to give correct results which this model predicts. The thermal and mechanical properties of the material used for such temperature changes are of the order of $5 \%$ and are considered constant.

\section{A. Calculation of the dimensionless parameters}

The intercepting block is made from a Copper alloy, namely $\mathrm{CuZrCr} 18150$. Its chemical composition is $98.9 \%$ copper, $1 \%$ zirconium and $0.11 \%$ chromium. The material properties are given in Table I and the power deposition parameters in Table II. The stresses in the perfect sphere will depend greatly on the width of the Gaussian which in turn is directly related to the amplitude $(A)$ and gradients of the source term. Two models are considered in Table II both allowing for spherically symmetric solutions. Namely a geometric average model and a conservative model. The conservative model uses the smallest $\sigma$ component as the power deposition width. The geometric average model uses $\sigma=\left(\sigma_{1} \sigma_{2} \sigma_{3}\right)^{1 / 3}$. Let $\sigma_{V M_{\text {ave }}}, \sigma_{V M_{\text {asy }}}$ and $\sigma_{V M_{\min }}$ be Von Mises stresses of the geometric average model, the asymmetric Gaussian beam model and the minimum width beam model, respectively.

From the linearity of equation (1) one can construct a fit of an asymmetric power source. This is done using a linear combination of spherically symmetric Gaussian

TABLE I. Parameters of the beam intercepting material presented in the nomenclature. All values are in SI units and at room temperature.

\begin{tabular}{lcccccc}
\hline \hline$\rho$ & $\lambda$ & $\mu$ & $c$ & $c_{0}$ & $\alpha$ & $k$ \\
\hline $8.89 \times 10^{3}$ & $2.93 \times 10^{10}$ & $5.17 \times 10^{10}$ & 383 & $3.8 \times 10^{3}$ & $16.7 \times 10^{-6}$ & 323 \\
\hline \hline
\end{tabular}


TABLE II. Energy deposition parameters retrieved from FLUKA simulations [2]. $A$ is the peak of the power density deposition calculated as follows: if $P=9.9238 \times 10^{9}$ is the beam instantaneous power then it is equal to the integral of $g(r)=$ $A \exp \left(-r^{2} / 2 \sigma^{2}\right)$ over the whole space. This is equal to $A * 2 \sqrt{2} \sigma^{3} \pi^{1.5}$, so that $A=P /\left(2 \sqrt{2} \sigma^{3} \pi^{1.5}\right) . \sigma$ is the standard deviation of the assumed Gaussian beam. It is calculated by fitting the energy deposition curve along $\mathrm{x}, \mathrm{y}$ and $\mathrm{z}$ in order to get $\sigma_{x}, \sigma_{y}$ and $\sigma_{z}$. From this we obtain $\sigma=\left(\sigma_{1} \sigma_{2} \sigma_{3}\right)^{1 / 3}$ and $\sigma=\sigma_{1}$ for the rows 1 and 2 respectively. $t_{p}$ is the pulse length and $L$ the characteristic length of the system (the distance of the peak of energy deposition to the closest surface of the BID). All values are in SI units.

\begin{tabular}{lcccc}
\hline \hline Assumption & $A$ & $\sigma$ & $t_{p}$ & $L$ \\
\hline Average & $5.64 \times 10^{14}$ & 0.0104 & $1 \cdot 0.94 \times 10^{-6}$ & 0.0465 \\
Conservative & $2.92 \times 10^{15}$ & 0.006 & $1 \cdot 0.94 \times 10^{-6}$ & 0.0465 \\
\hline \hline
\end{tabular}

distributions. Which in turn would enable a solution in the form of a linear combination of spherically symmetric $\Phi$ 's which can then yield a Von Mises stress at a desired precision depending on the precision of the fit.

Back to the spherical models, the dimensionless parameters computed from Tables I and II are presented in Table III for both models.

In order to use the above models for thermal stresses the nondiffusive assumption is verified using the peak temperature ratio defined in [22].

\section{B. Results using the analytical solution}

From Fig. 1, the peak of stress occurs at $r_{\max } \sim 1.42$ which is found in the ideal region since $1.414<\Pi_{2}-\Pi_{4}$. Therefore our model is valid.

For the geometric average model $\Pi_{4}=0.344$, Eq. (24) can be approximated by the asymptotic solution (see Fig. 2) and gives the value of the peak of the Von Mises stress: $\sigma_{V M_{\text {ave }}}^{\max }=2 \mu \cdot \Pi_{c} \cdot 0.1224 \Pi_{4}^{2} \simeq 5.8 \mathrm{MPa}$. This value is well below the proportional limit strength of this alloy $(\sim 200 \mathrm{MPa})$.

In the case of the conservative model maximum stresses are expected to be much higher and equate to $\sigma_{V M_{\min }}^{\max } \simeq 80 \mathrm{MPa}$, still less than the proportional limit. The latter Von Mises stress value is within the orders of

TABLE III. Nondimensional parameters obtained from Tables I and II. The first and second rows correspond to different energy deposition width considerations, namely one that uses a geometric average and one that uses the minimum width of the $3 \mathrm{D}$ Gaussian distribution fit.

\begin{tabular}{lcccc}
\hline \hline Assumption & $\Pi_{2}$ & $\Pi_{4}$ & $\Pi_{6}$ & $\Pi_{c}$ \\
\hline Average & 4.48 & 0.344 & 0.165 & $3.75 \times 10^{-3}$ \\
Conservative & 7.75 & 0.595 & 0.284 & $1.94 \times 10^{-2}$ \\
\hline \hline
\end{tabular}

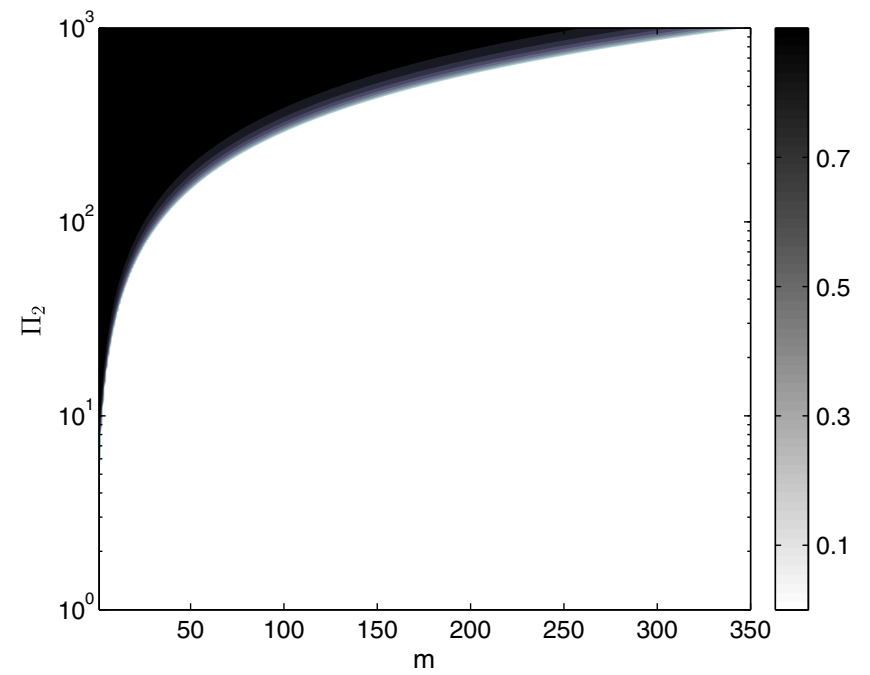

FIG. 6. Contour plot of $\epsilon_{\text {rms }}$ versus the number of terms in the series $m$ on the abscissa and $\Pi_{2}$ in log-log scale on the ordinate.

magnitude of full finite element method studies (60 MPa) in [4].

\section{Results using the series solution}

The series solution is used to verify the analytical solution results. The relation of the precision of the series solution to the truncation of the series is illustrated in Figure 6. Figure 7 shows an excellent match between the bounded medium solution and the infinite medium

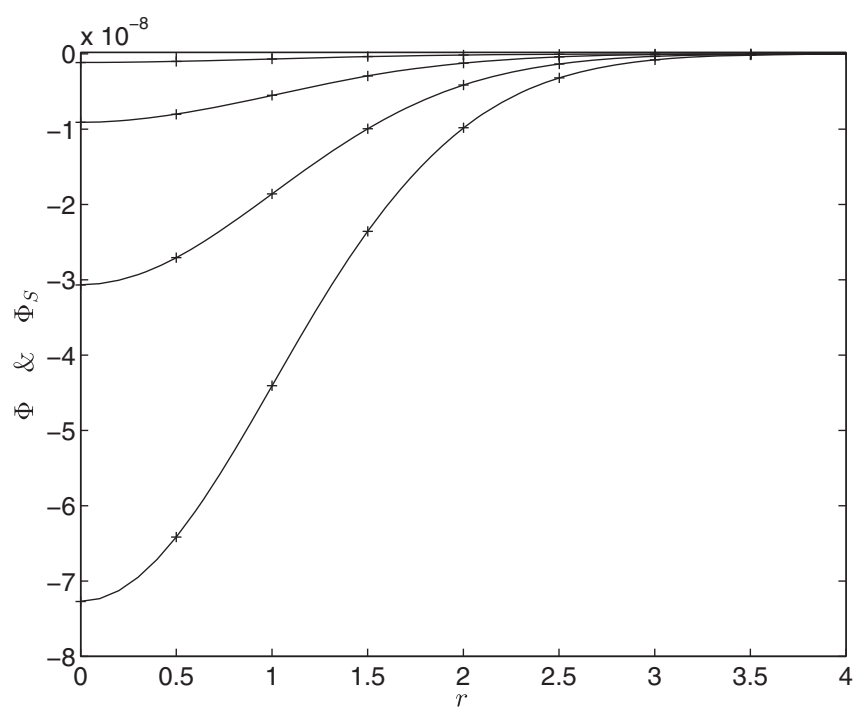

FIG. 7. Plot of the exact infinite medium solutions versus the bounded medium series solutions under Dirichlet boundary conditions. The infinite medium solutions, in solid, are using the parameters shown in Table I. The truncated series solutions are in cross points, with $m=200$ yielding an $\epsilon_{\mathrm{rms}} \sim 10^{-4}$. The four distinct curves shown in descending order are for $t=0.25$, $0.5,0.75,1$ respectively. 


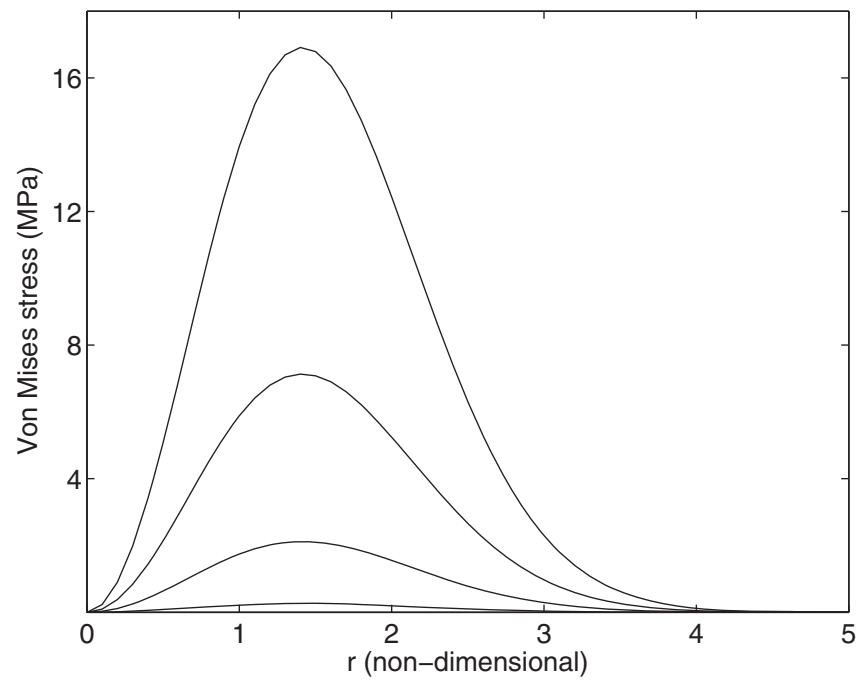

FIG. 8. Plot of the Von Mises stress versus the radius for the beam and material setup described in Tables I and II. In ascending order the curves drawn are for times of $t=0.25,0.5,0.75,1$, respectively.

solution. The truncated series solution for the finite field is computed using $m=200$ terms and yields an error of $\epsilon_{\mathrm{rms}} \sim 10^{-4}$ defined by Eq. (39). From Fig. 5 the dynamic effects are expected to be important and the model cannot be simplified using its quasistatic counterpart. The position of the peak of stress is far from that of quasistatic elasticity and the error defined in Eq. (30) is $E_{\text {err }}=0.9899 \sim 1$ which makes the quasistatic solution in this case meaningless.

Figure 8 shows the Von Mises stresses for different times $t$ including for $t=1$ where maximum stress is reached. The maximum stress position is verified using the algorithm presented in Sec. IV E. These results match those found by the analytical solution.

\section{CONCLUSIONS}

We obtain closed form solutions for the uncoupled thermoelastic problem in infinite media under a Gaussian spherical source. The spherical Gaussian is a model of a beam impacting a material. The diffusivity of the material is assumed negligible such that the temperature field can easily be obtained from the energy source field. The problem is nondimensionalized and two nondimensional parameters are used to verify the necessary assumption of negligible heat diffusion during the pulselength. The domain of applicability of such solutions is well defined using intervals of the nondimensional $\Pi_{i}$ parameters. The solutions to the elasticity equations yield the compressive potential, the displacements and the stresses-note that the shear potential is nil since the source is a gradient. The elegant form of the solutions allows one to optimize any applicable problem without the iterative and lengthy process necessary in numerical simulations.

An important point is raised as to the position of the maximum occurring stress and its value with respect to the dimensionless parameters. In the presented case study the aforementioned solutions are compared with their finite geometry counterparts only to obtain very similar results. The relative errors presented in the case study are in accordance with the figures presented in the theory section. The present model yields excellent results in the elastic regime.

Finally, taking advantage of the linearity of the Navier equations, one can fit an asymmetric source with a sum of Gaussian distributions in order to get precise quantitative results. Assuming the validity of our models for each Gaussian, one could bypass the use of any finite element simulations in addition to being several order magnitude faster than conventional finite element simulations.

\section{ACKNOWLEDGMENTS}

The authors thank Richard Craster for many useful inputs and Federico Carra for providing accurate data used to illustrate the underlying theory.

[1] N. J. Carron, An Introduction of the Passage of Energetic Particles through Matter (Taylor and Francis Group, Boca Raton Florida, 2007).

[2] G. Battistoni F. Cerutti, A. Fassò, A. Ferrari, S. Muraro, J. Ranft, S. Roesler, and P. R. Sala, The FLUKA code: Description and benchmarking, AIP Conf. Proc. 896, 31 (2007).

[3] A. Fasso, A. Ferrari, J. Raft, and PR. Sala, in Advanced Monte Carlo for Radiation Physics, Particle Transport Simulation and Applications (Springer, Lisbon, Portugal, 2001), p. 955.

[4] A. Perillo-Marcone et al., in Proceedings of the 4th International Particle Accelerator Conference, IPAC2013, Shanghai, China, 2013 (JACoW, Shanghai, China, 2013), p. 3436.

[5] A. Sarrio, F. Loprete, and C. Maglioni, in Proceedings of the 3rd International Particle Accelerator Conference, New Orleans, LA, 2012 (IEEE, Piscataway, NJ, 2012), p. 3761 .

[6] B. Goddard, A. Presland, W. Weterings, and L. Massidda, in Proceedings of the 10th European Particle Accelerator Conference, Edinburgh, Scotland, 2006 (EPS-AG, Edinburgh, Scotland, 2006), p. 1511.

[7] M. Scapin, L. Peroni, and A. Dallocchio, Damage evaluation in metal structures subjected to high energy deposition due to particle beams, J. Phys. Conf. Ser. 305, 012062 (2011).

[8] N. A. Tahir, R. Schmidt, and M. Brugger, Interaction of super proton synchrotron beam with solid copper target: Simulations of future experiments at hiradmat facility at CERN, Nucl. Instrum. Methods Phys. Res., Sect. A 606, 186 (2009). 
[9] A. Ijspeert, R. Bellone, and P. Sievers, The new internal beam dump at the sps. temperature and stress calculations and its design, IEEE Trans. Nucl. Sci. 28, 3062 (1981).

[10] L. G. Hector and R. B. Hetnarski, Thermal stresses due to a laser pulse: Elastic solution, J. Appl. Mech. 63, 38 (1996).

[11] M. Lax, Temperature rise induced by a laser beam, J. Appl. Phys. 48, 3919 (1977).

[12] M. Lax, Temperature rise induced by a laser beam ii. the non-linear case, Appl. Phys. Lett. 33, 786 (1978).

[13] L. P. Welsh, J. A. Tuchman, and I. P. Herman, The importance of thermal stresses and strains induced in laser processing with focused Gaussian beams, J. Appl. Phys. 64, 6274 (1988).

[14] I. C. Sheng and Y. Cheng, Thermoelastic analysis for a semi-infinite plane subjected to a moving Gaussian heat source, Journal of Thermal Stresses 14, 129 (1991).

[15] P. Sievers, Elastic stress waves in matter due to rapid heating by an intense high-energy particle beam, CERN, 1974 (unpublished).

[16] W. Stein, L. E. Ahle, and D. L. Conner, in Proceedings of the 21st Particle Accelerator Conference, Knoxville, TN, 2005 (IEEE, Piscataway, NJ, 2005), p. 2185.

[17] W. Weterings, T. Antonakakis, B. Balhan, J. Borburgh, B. Goddard, C. Maglioni, and R. Versaci, in Proceedings of the 3rd International Particle Accelerator Conference, New Orleans, LA, 2012 (IEEE, Piscataway, NJ, 2012), p. 556.

[18] O. Jakel, State of the art in hadron therapy, AIP Conf. Proc. 958, 70 (2007).

[19] W. P. Levin, H. Kooy, J. S Loeffler, and T. F. DeLaney, Proton beam therapy, Br. J. Cancer 93, 849 (2005).

[20] H. Paganetti, Proton Therapy Physics (CRC Press, Boca Raton, Florida, 2012).

[21] W. Nowacki, Dynamic Problems of Thermoelasticity (Noordhoff International Publishing, Leyden, The Netherlands, 1975).

[22] T. Antonakakis, C. Maglioni, and V. Vlachoudis, Closed form solutions of the heat diffusion equation with a Gaussian source, Int. J. Heat Mass Transfer 62, 314 (2013).

[23] G. Barton, Elements of Green's Functions and Propagation (Oxford University Press, New York, 1989).

[24] G. Dieter, Mechanical Metallurgy (McGraw-Hill, London, 1988).

[25] MATLAB, www.mathworks.com, 2012.

[26] R. Haberman, Elementary Applied Partial Differential Equations (Prentice Hall, Englewood Cliffs, New Jersey, 1987). 